\title{
Review
}

\section{Regulation of AMPK by the Ubiquitin Proteasome System}

\author{
Makhosazane Zungu, ${ }^{*}$ Jonathan C. Schisler, ${ }^{\dagger}$ \\ M. Faadiel Essop, ${ }^{\ddagger}$ Chris McCudden, ${ }^{\S}$ \\ Cam Patterson, ${ }^{\dagger}$ and Monte S. Willis ${ }^{\dagger, \S}$ \\ From the Discipline of Human Physiology,* University of KwaZulu \\ Natal, Durban, South Africa; the McAllister Heart Institute, ${ }^{\dagger}$ and the \\ Department of Pathology \& Laboratory Medicine, ${ }^{\S}$ University of \\ North Carolina, Chapel Hill, North Carolina; and the \\ Department of Physiological Sciences, ${ }^{\star}$ Stellenbosch University, \\ Stellenbosch, South Africa
}

The 5'-AMP-activated protein kinase (AMPK) functions as a metabolic fuel gauge that is activated in response to environmental stressors to restore cellular energy balance. In the heart, AMPK coordinates the activation of glucose and fatty acid metabolic pathways to ensure increased production of myocardial ATP when required, such as during cardiac ischemia/reperfusion and hypertrophy, causing an increase in AMPK activity that can be viewed as both protective and maladaptive. While we understand the basic regulation of AMPK activity by kinases, recent studies have introduced the concept that AMPK is regulated by other post-translational modifications, specifically ubiquitination. These studies reported that the ubiquitin ligase cell death-inducing DFFA-like effector a ubiquitinates the $\beta$ subunit of AMPK to regulate its steady-state protein levels. Other investigators found that AMPK regulatory components, including the AMPK $\alpha$ subunit and AMPK kinases NUAK1 and MARK4, can be ubiquitinated with atypical ubiquitin chains. The USP9X-deubiquitinating enzyme was identified to remove ubiquitination from both NUAK1 and MARK4. Lastly, AMPK activation increases the expression of the ubiquitin ligases MAFBx/Atrogin-1 and MuRF1. These ubiquitin ligases regulate key cardiac transcription factors to control cardiomyocyte mass and remodeling, thus suggesting another mechanism by which AMPK may function in the heart. The relevance of AMPK ubiquitination in cardiac disease has yet to be tested directly, but it likely represents an important mechanism that occurs in common cardiac diseases that may be targeted for therapy. (Am J Pathol 2011, 178:4-11; DOI: 10.1016/j.ajpath.2010.11.030)
The 5'-AMP-activated protein kinase (AMPK) functions as a metabolic fuel gauge that is activated in response to numerous environmental stressors to restore cellular and whole-body energy balance. ${ }^{1,2}$ AMPK is allosterically regulated by the competitive binding of AMP and ATP, thereby "sensing" cellular energy status and, on activation, triggers compensatory ATP-generating mechanisms while attenuating ATP-consuming processes. ${ }^{3}$ Perturbations in cardiac metabolism are closely linked to the onset and progression of cardiovascular diseases; given the central role of AMPK in regulating cellular energetics, there is considerable interest in the determination of the precise role(s) of AMPK in cardiac pathophysiological states and evaluation of the utility of modulating AMPK activity as a therapeutic intervention. ${ }^{4}$ This review discusses AMPK function in normal and diseased hearts, with emphases on AMPK and protein degradation via the ubiquitin proteasome pathway-a potential novel approach to treating cardiovascular disease. These are significant new findings as ubiquitination is emerging as a pivotal regulatory mechanism that rivals phosphorylation in its overall significance in biology. ${ }^{5}$

\section{Structure and Regulation of AMPK}

AMPK is a highly conserved heterotrimeric enzyme consisting of three subunits, $\alpha, \beta$, and $\gamma$, with multiple genes encoding distinct subunit isoforms (ie, $\alpha 1, \alpha 2, \beta 1, \beta 2, \gamma 1, \gamma 2$, and $\gamma 3$ ). The structure and regulation of AMPK is the subject of an extensive recent review ${ }^{6}$; therefore, this article presents a brief structural and regulatory overview and focuses on the heart-specific aspects of AMPK. The $\alpha$ subunit of AMPK contains the catalytic domain and the Thr 172 residue targeted by upstream kinases required for subsequent activation. ${ }^{7}$ The $\beta$ subunit contains a glycogen-binding do-

Supported by the National Heart, Lung, and Blood Institute grant R01HL065619 (C.P.) and the American Heart Associations (Scientist Development Grant) (M.S.W.).

M.Z. and J.C.S. contributed equally to this manuscript.

Accepted for publication August 13, 2010

Address reprint requests to Monte S. Willis, M.D., Ph.D., McAllister Heart Institute, Department of Pathology \& Laboratory Medicine, University of North Carolina, Medical Biomolecular Research Building, Rm 2336, 103 Mason Farm Road, Chapel Hill, NC 27599-7525. E-mail: monte_willis@med. unc.edu. 
main that is important for kinase function and substrate definition ${ }^{8}$ and functions as scaffold for $\alpha$ and $\gamma$ subunit binding. ${ }^{9}$ The $\gamma$ subunit consists of four tandem or cystathione $\beta$-synthase (CBS) repeats, forming two basic functional units called Bateman domains that bind AMP or ATP in a mutually exclusive manner, depending on the particular intracellular energetic milieu. ${ }^{10,11}$

The $\alpha 1$ and $\beta 1$ isoforms are ubiquitously expressed in mammals, whereas the $\alpha 2$ and $\beta 2$ isoforms are enriched in the heart as well as skeletal muscle. ${ }^{12,13}$ Of the three $\gamma$ subunits, $\gamma 1$ is ubiquitously expressed, ${ }^{14}$ whereas $\gamma 2$ is enriched in the heart (but not skeletal muscle), and $\gamma 3$ is expressed exclusively in skeletal muscle. ${ }^{15}$ Together, these studies demonstrate distinct expression patterns for AMPK subunits; for example, the cardiac AMPK multi-protein complex predominantly consists of $\alpha 2-\beta 2-\gamma 2$ subunits, while $\alpha 2-\beta 2-\gamma 3$ is the major complex found in skeletal muscle. ${ }^{14}$ It is likely that different AMPK subunit isoform combinations play different roles in various tissues, depending on the prevailing intracellular energetic status; however, this interesting possibility requires further study.

AMPK activation is dependent on the cellular AMP/ATP ratio and the phosphorylation status of $\alpha$ Thr 172 that continually cycles between phosphorylated and dephosphorylated. ${ }^{16}$ Several cardiac stressors, such as ischemia, result in increased AMP and decreased ATP levels. ${ }^{17}$ With a rise in the AMP/ATP ratio, AMP displaces the ATP bound to the $\gamma$ regulatory subunit, leading to three different functional outcomes. ${ }^{3,14}$ First, AMP allosterically activates AMPK activity by a factor of 10 . Additionally, AMP binding causes a conformational change, thereby enhancing access by upstream AMPK kinases (AMPKK) and further increasing AMPK activation ${ }^{18}$ and relieving the inhibition of the autoinhibitory domain. ${ }^{19}$ Last, AMP binding inhibits $\alpha$ Thr 172 dephosphorylation by upstream phosphatases, thus promoting increased AMPK phosphorylation. ${ }^{20}$

However, there is also AMP/ATP-independent activation of cardiac AMPK by upstream AMPKKs, such as the serine-threonine liver kinase $\mathrm{B} 1$ (LKB1) and $\mathrm{Ca}^{2+} / \mathrm{cal}-$ modulin-dependent protein kinase kinase (CAMKK) 21,22 as well as the hormones insulin ${ }^{23-25}$ and adiponectin. 26,27 LKB1 is highly expressed in the heart and is thought to be constitutively active under basal conditions. ${ }^{4}$ Accordingly, LKB1-deficient mouse hearts are unable to activate cardiac AMPK $\alpha 2 .{ }^{22}$ CAMKK is expressed at relatively low levels in the heart and is triggered by an increase in cytosolic $\mathrm{Ca}^{2+}$ levels and subsequently activates AMPK without altered AMP/ATP ratios. ${ }^{21}$ Transforming growth factor- $\beta$ (TGF- $\beta$ )-activated kinase- 1 is also expressed in the heart and activates AMPK, although its role in this process is less well understood. ${ }^{28}$

Interestingly, well-described cardiometabolic drugs, such as metformin and statins, can also activate cardiac AMPK, although likely through indirect means. ${ }^{29}$ For example, it has been suggested that metformin inhibits mitochondrial respiratory chain complex I, increasing the AMP/ATP ratio and subsequent AMPK activation. ${ }^{30,31}$ However, others have found that metformin can activate AMPK independent of changes in the AMP/ATP ratio. ${ }^{32}$ This discrepancy may be explained by differences in metformin dosing and length of exposure; further studies are required to elucidate the precise mechanism of metformin on cardiac AMPK activity. AICA riboside (AICAR) is an adenosine analog that allosterically activates AMPK and is often used in experimental studies; however, the short half-life of AICAR and effects on glycemic regulation limits AICAR as an effective agent for long-term activation of AMPK in vivo. ${ }^{29}$

\section{AMPK Function in the Heart}

The normal mammalian heart has tremendous energy requirements; as such, cardiac fuel substrate selection is a complex process that is orchestrated by a number of factors that include circulating metabolite and hormone levels, myocardial substrate uptake rates, and intracellular signaling cascades that regulate metabolic pathway flux at multiple levels. ${ }^{33}$ AMPK impacts cardiac metabolism at multiple levels: (1) increasing glucose metabolism through increased glucose transport and activation of phosphofructokinase (PFK); (2) enhancing mitochondrial fatty acid $\beta$-oxidation (FAO) by increasing fatty acid uptake and phosphorylating acetyl-CoA carboxylase (ACC), a key regulator of fatty acid oxidation; (3) increasing mitochondrial biogenesis by activation of peroxisome proliferator-activated receptor- $\gamma$ co-activator- $1 \alpha$ (PGC- $1 \alpha$ ), a key transcriptional modulator of mitochondrial biogenesis ${ }^{34}$; and (4) inhibiting energy-consuming pathways, such as the mammalian target of rapamycin (mTOR) and downstream targets p70S6K and 4EBP-1, decreasing cardiac protein synthesis. ${ }^{35}$ Together, AMPK activation of myocardial glucose and fatty acid metabolic pathways are coordinated at multiple levels: fuel substrate availability, uptake, and breakdown, ultimately ensuring increased production of myocardial ATP when required.

Metabolic derangements are closely linked to the onset of numerous cardiac pathophysiologies. Accordingly, a multitude of studies focus on understanding the role of AMPK under such conditions, such as myocardial ischemia-reperfusion (I/R), cardiomyopathies, and cardiac hypertrophy. The main observations of these studies are highlighted in Table 1. 14,22,26,36-49

\section{Regulation of AMPK by Post-Translational Modification of Ubiquitin}

Post-translational modification of proteins is the process by which chemical modifications are made to a protein after it is translated. There is a wide range of post-translational modifications that can be made to proteins. One of the best-studied modifications is phosphorylation, whereby enzymes add a phosphate group to a protein, often at specific serine, threonine, or tyrosine residues, to change that protein's activity or fate. Phosphorylation of the $\alpha$ subunit of AMPK is the best-delineated system of AMPK regulation to date. However, several recent studies have implicated the modification of AMPK with ubiquitin, suggesting another layer of AMPK regulation in the heart. With many reports demonstrating a significant role of AMPK in heart disease (Table 14,22,26,36-49) and our increasing appreciation of the ubiquitin proteasome system in the heart (for a comprehensive review, see Ro- 
Table 1. The Role of AMPK in Cardiac Disease Models

\begin{tabular}{|c|c|}
\hline Ischemia-reperfusion & $\begin{array}{l}\text { Observational events in mouse models } \\
\text { Onset of ischemia increases AMPK activity }{ }^{36} \\
\text { Corresponding increases in GLUT4 translocation, PFK2 activity, and glycolytic ATP } \\
\text { production }{ }^{37} \\
\text { Increase in AMPKK activity } 22,38 \\
\text { Cardio-protective role for AMPK } \\
\text { Some genetic mouse models support a beneficial role for AMPK activation during } \\
\text { ischemia-reperfusion: } \\
\text { Decreased cardiomyocyte death and infarct size, maintain cardiac function }{ }^{26,37,39} \\
\text { Ischemic preconditioning increases AMPK activity }{ }^{40} \\
\text { Maladaptive role for AMPK } \\
\text { AMPK activation also increases fatty acid oxidation, leading to detrimental consequences: } \\
\text { Oxygen wasting } \\
\text { Mitochondrial uncoupling } \\
\text { Uncoupling of glycolysis from glucose oxidation } \\
\end{array}$ \\
\hline PRKAG2 cardiac syndrome & $\begin{array}{l}\text { Mutations within human AMPK } \gamma 2 \text { gene (PRKAG2) } \\
\text { Ventricular pre-excitation, progressive conduction system disease, hypertrophy }{ }^{43} \\
\text { Excessive glycogen deposition }{ }^{14} \\
\text { Human phenotype supported by multiple mouse models } \\
\text { Excessive glycogen accumulation and hypertrophy }{ }^{44,45} \\
\text { Glycogen accumulation may cause ventricular pre-excitation and/or conduction } \\
\text { deficiencies }{ }^{43,46,47}\end{array}$ \\
\hline Pathological cardiac hypertrophy & $\begin{array}{l}\text { There are conflicting reports as to the role of AMPK activation in response to pathological } \\
\text { hypertrophy stimuli: } \\
\text { Isoform-specific changes in AMPK expression and increases in AMPK activity occur } \\
\text { during pressure overload in mice }{ }^{48} \\
\text { In isolated cardiomyocytes, activation of AMPK decreased protein synthesis during } \\
\text { phenylephrine-stimulated and Akt-stimulated hypertrophy, suggesting AMPK activation } \\
\text { may prevent the progression of cardiac hypertrophy }{ }^{49}\end{array}$ \\
\hline
\end{tabular}

Gene encoding the 5'-AMP-activated protein kinase subunit gamma-2 (which makes up AMPK). Glut4, glucose transporter 4; PFK2, phosphofructokinase 2; PRKAG2, protein kinase, AMP-activated, gamma-2 non-catalytic subunit.

driguez et al $2009^{50}$ ), the significance of AMPK ubiquitination is just beginning to be appreciated.

\section{Ubiquitin Proteasome System}

Post-translational modification of proteins with ubiquitin is a multistep enzymatic process that reacts to the carboxylic acid of the ubiquitin to protein lysines to form a covalent amide bond (Figure 1A). The small protein ubiquitin is first activated in a two-step process. First, the E1 ubiquitin-activating enzyme interacts with ubiquitin in an ATP-dependent process to form a thioester linkage between the C-terminal carboxyl group of ubiquitin and the E1 sulfhydryl moiety. Ubiquitin is then transferred to the active cysteine of the E2 ubiquitin-conjugating enzyme. The specificity of the ubiquitination process is found in the E3 ubiquitin ligases. E3 ubiquitin ligases function to recognize specific substrates and to transfer ubiquitin to their final lysine on target proteins (Figure 1A). There are hundreds of ubiquitin ligases that have been identified, with at least nine identified in the heart to date. ${ }^{50}$ The single $\mathrm{E} 1$ is able to bind to dozens of E2s, which bind hundreds of E3s to regulate a host of cellular processes. After the addition of a single ubiquitin, additional ubiquitin molecules can be added to form a polyubiquitin chain. Ubiquitin has seven lysine residues by which this can occur [ie, Lys 6, Lys 11, Lys 27, Lys 29, Lys 33, Lys 48, and Lys 63 (Figure 1B)]. The more common chains that have been described to date are through the lysine 48 , which are recognized by the $26 \mathrm{~S}$ proteasome (Figure 1A). Polyubiquitinated chains can be removed by a family of enzymes called de-ubiquitinating enzymes (DUBs), consisting of a large group of proteases that counter the ubiquitin-dependent metabolic pathways by cleaving the bonds between ubiquitin and proteins. The human genome encodes approximately 100 DUBs specific for ubiquitin. ${ }^{51}$ DUBs function to recycle and proofread protein ubiquitination and act in the disassembly of chains that inhibit protein activity.

While the canonical polyubiquitin (lys48) chains target their substrates for degradation, linkages through the other available lysines, including the N-terminus, ${ }^{52}$ and branched chains containing multiple linkage types ${ }^{53}$ also occur. These atypical chains generally do not target proteins for degradation, but they do play important roles in regulating cellular processes. ${ }^{54}$ Atypical ubiquitination is most studied in nuclear factor $\kappa \mathrm{B}$ signaling, receptor endocytosis, and DNA repair processes ${ }^{55,56}$; therefore, it is not surprising that atypical ubiquitination regulates AMPK.

\section{Emerging Concept: AMPK Is Regulated by Ubiquitin}

Recent studies identified both direct and indirect mechanisms by which the ubiquitin proteasome system regulates AMPK activity. While LKB1 is the best-characterized AMPK kinase, there are others that regulate the activity of AMPK, including the protein NUAK1 (AMPK-related kinase 5) and MARK4 (microtubule-affinity-regulating kinase 4). Recent studies demonstrated that the de-ubiquitinating enzyme USP9X interacts with endogenous NUAK1 and MARK4 in HEK293 cells by immunoprecipi- 

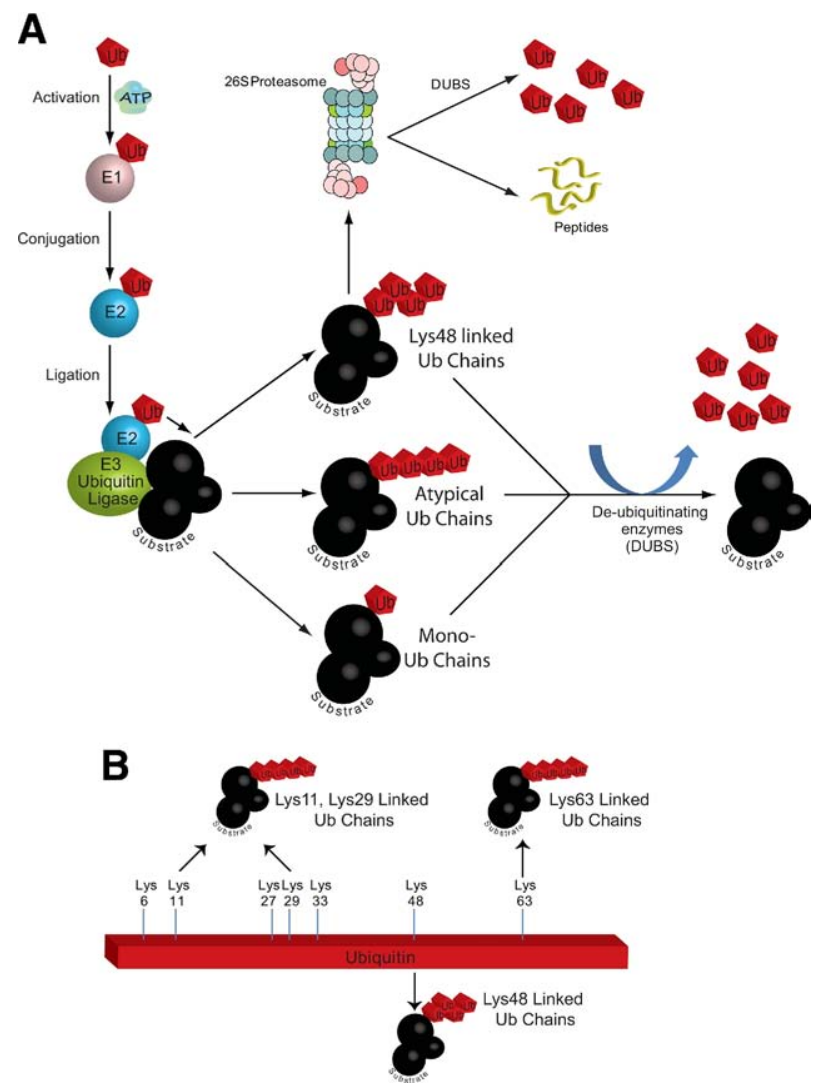

Figure 1. The ubiquitin proteasome system. A: Specific proteins are recog nized by E3 enzymes (ubiquitin ligases) and ubiquitinated in a multistep process. The E1 enzyme activates ubiquitin, which is then transferred to one of the dozens of E2 enzymes. The E3 then catalyzes the transfer of the ubiquitin from the E2 to the recognized substrate in an ATP-dependent manner. Ubiquitinated substrates with canonical Lys 48 chains are then recognized by the $26 \mathrm{~S}$ proteasome and targeted for degradation or de-ubiquitinated as part of the ubiquitin proteasome system regulation and ubiquitin chain quality control. Substrate activity and/or location may be altered if mono-ubiquitin or atypical polyubiquitin chains are added. B: Polyubiquitin chains can be formed by any of the seven lysines within ubiquitin as well as by the N-terminal lysine to form atypical (Lys11, Lys29, and Lys63), canonical (Lys48), or linear (N-terminal lysine) chains. Our understanding of the significance of all of the non-canonical chains is incomplete.

tation. ${ }^{57}$ The AMPK kinases NUAK1 and MARK4 are also polyubiquitinated in vivo. ${ }^{57}$ Inhibiting the deubiquitinating enzyme enhances NUAK and MARK4 ubiquitination, implicating the role of USP9X in ubiquitinating these AMPK kinases in vitro. ${ }^{57}$ In vitro deubiquitination studies confirmed the ability of USP9X to directly deubiquitinate NUAK1 and MARK4; conversely, knock-down of USP9X in vitro enhanced their ubiquitination. ${ }^{57}$ Expressing mutant NUAK and MARK4 constructs unable to interact with the USP9X DUB causes hyperubiquitination of NUAK and MARK4, resulting in decreased AMPK catalytic activity. ${ }^{57}$ Ubiquitin-dependent inhibition of AMPK kinase activity is thought to be due to the interference of ubiquitin with phosphorylation of the activation (T-loop) residues. ${ }^{57}$ The polyubiquitination of NUAK and MARK4 is formed predominantly by linkages at lys29 and lys33, but not other lysines, indicative of atypical polyubiquitin chains. ${ }^{57}$ The mechanisms by which ubiquitination regulates the NUAK and MARK4 AMPKKs, which then affect AMPK activity, are summarized in Figure 2. ${ }^{58}$

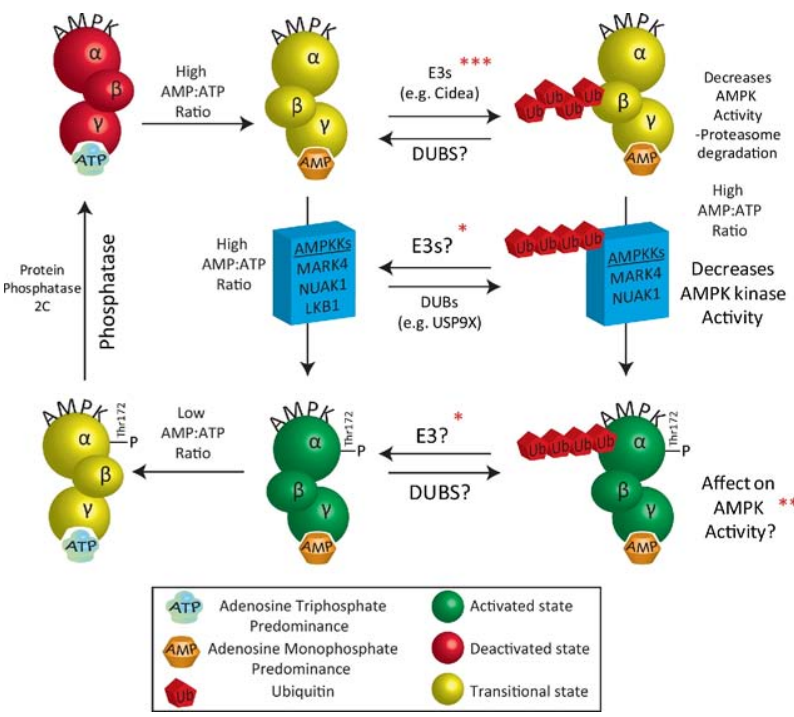

Figure 2. Post-translational regulation of AMPK activity. The AMP-activated protein kinase (AMPK) is a metabolite-sensing serine-threonine kinase that acts as a master regulator of cellular energy metabolism, with the ability to regulate lipid, glucose, and protein metabolism in response to decreasing ATP levels. The AMPK holoenzyme-consisting of $\alpha, \beta$, and $\gamma$ subunits-is regulated by AMP:ATP ratios, whereby AMP binds the Bateman (CBS) domains in the $\gamma$ subunit, resulting in a conformational change which allows the $\alpha$ catalytic domain to be phosphorylated (Thr172) by one of many AMPK kinases. Activation (green) and deactivation (red) has canonically been described by AMPK kinases and phosphatases (left column). Protein phosphatases, such as protein phosphatase 2C, also counteract AMPK. ${ }^{58}$ The role of the ubiquitin proteasome system in regulating AMPK activity is just beginning to be understood (right column). Polyubiquitination and proteasome-dependent degradation has been reported to be mediated by the E3 ubiquitin ligase Cidea (top, right), which inhibits downstream AMPK activity. The AMPK kinases MARK4 and NUAK1 are de-ubiquitinated by the USP9X DUB, affecting the ability of these enzymes to activate AMPK (middle, right). Last, atypical ubiquitin chains can be placed on the $\alpha$ subunit of AMPK have been described, and that may affect its activity. *A DUB has been identified, although competing E3 not identified (See text for details). ** Ubiquitination of the alpha1 subunit of AMPK is enhanced in the presence of a DUB inhibitor (See text for details). ${ }^{* * * *}$ Cidea interacts with the beta subunit of AMPK to target (polyubiquitinate) it for proteasome-mediated degradation (See text for details).

These fascinating studies raise many additional questions about how the ubiquitin proteasome system regulates AMPK activity. Most of the work to date on the ubiquitin proteasome system has focused on the E1, E2, and particularly the E3 ubiquitin ligases that regulate substrate activities. Much less focus has been put on identifying de-ubiquitinases and their role in regulating cellular processes (recently reviewed ${ }^{59-61}$ ). However, the therapeutic potential of deubiquitinating enzyme inhibitors has been a new line of investigation in cancer chemotherapies. $^{62}$ So it is interesting that a specific DUB has been identified in regulating AMPK kinases without the identification of the E3 ubiquitin ligases they are competing with (Figure $2^{58}$ ). Moreover, the alpha1 subunit of AMPK is found to be ubiquitinated in the presence of DUB inhibitors (Figure $2^{58}$ ). In the presence of NEM, an inhibitor of de-ubiquitination, enhanced polyubiquitination of immunoprecipitated AMPK alpha1 subunit has been observed. ${ }^{57}$ Interestingly, the polyubiquitin chains on this subunit were predominantly atypical (lys29 and lys33) linkages. This observation indicates an important role for DUBs in the direct regulation of AMPK and raises the question of what E3 ubiquitin ligase(s) are placing the 
atypical polyubiquitin chains on the AMPK alpha1 subunit. It also remains to be determined if this modification affects AMPK activity.

To date, only one study has identified an E3 ubiquitin ligase that mediates the ubiquitination and degradation of AMPK. In brown adipose tissue, the Cidea (cell deathinducing DFFA-like effector a) protein ubiquitinates and degrades AMPK. ${ }^{63}$ Cidea is found in many tissues, including heart, and is found to regulate metabolism through its interaction with AMPK. Cidea co-localizes in the endoplasmic reticulum and forms a complex with AMPK in vivo. ${ }^{63}$ Cidea specifically interacts with the $\beta$ subunit of AMPK, but not the $\alpha$ or $\gamma$ (Figure $2^{58}$ ). ${ }^{63}$ When Cidea is co-expressed with AMPK $\beta$ subunit, the stability of AMPK $\beta$ is reduced due to ubiquitin-dependent degradation. ${ }^{63}$ Cidea $^{-1-}$ mice have an enhanced AMPK stability and enzymatic activity, which is consistent with both the role of Cidea as an E3 ubiquitin ligase that regulates AMPK activity as well as with the increased energy expenditure and lean phenotype that could be explained by this enhanced AMPK activity. ${ }^{63}$ While the type of polyubiquitination that Cidea placed on the AMPK $\beta$ subunit was not identified in these studies, it is likely that the polyubiquitination is of the canonical (lys48)-type that targets proteins for proteasome-dependent degradation. This contrasts to the atypical lysine chains found on the $\alpha$ subunit in previous studies. ${ }^{63}$ However, atypical lys29 ubiquitin chains can also promote protein degradation via the lysosome, so parallels may exist between these two systems. ${ }^{64}$ Ubiquitination of AMPK by the ubiquitin ligase Cidea plays a role in inhibiting AMPK activity, likely by targeting the AMPK holo-enzyme or one of its subunits $(\beta)$ for degradation by the proteasome.

\section{AMPK Interacts with the Proteasome?}

Last, a recent study identified that AMPK interacts with the proteasome itself. In yeast two-hybrid studies, it has been reported that the PSMD11 proteasome subunit interacts with AMPK. ${ }^{65}$ This finding suggests that AMPK may physically interact with this proteasome subunit to affect its phosphorylation status and potential function. However, the effects of AMPK on the proteasome (and vice versa) have not been directly tested. With the realization of the importance of proteasome function in cardiac health and disease, these findings may prove to be significant as we better understand how AMPK regulates the proteasome, which may be important for its own (ie, AMPK's) activity described above.

\section{AMPK Activation Increases the Expression of Ubiquitin Ligases Relevant to Cardiac Disease}

While the regulation of AMPK activity by ubiquitination is just beginning to be elucidated, much more is known about how AMPK activation regulates the ubiquitination machinery, in particular the E3 ubiquitin ligases, the proteins that give specificity to the system and mediate the ubiquitination of specific substrates. This work has exclusively been performed in skeletal muscle. However, like many lines of study of the ubiquitin ligases MuRF1 and MAFBx/Atrogin-1, applicability to the heart is likely relevant.

\section{AMPK Activation Increases Expression of MuRF1 and MAFBx/Atrogin-1}

AMPK activation in striated muscle has dual effects. It enhances cellular processes involved in ATP production, including glucose uptake and fatty acid oxidation. ${ }^{66,67}$ It also inhibits processes that consume energy, such as protein synthesis. ${ }^{68-70}$ AMPK activation inhibits protein synthesis by decreasing the mTOR activation. ${ }^{68}$ Three studies have recently investigated how AMPK activation affects MuRF1 and MAFBx/Atrogin-1 expression, both in vitro and in vivo. They have generally found that AMPK activation increases MuRF1 and MAFBx/Atrogin-1 expression.

Stimulating C2C12 myotubes with AICAR (5-aminoimidazole-4-carboxamide 1- $\beta$-d-ribofuranoside) to enhance AMPK activity, Nakashima and Yakabe ${ }^{71}$ identified an enhanced protein degradation and increased MAFBX/ Atrogin-1 and MuRF1. In subsequent studies, Krawiec et $\mathrm{al}^{72}$ found that AICAR, metformin, or 2-deoxyglucose could all enhance MAFBx/atrogin-1and MuRF1 expression over time. Krawiec et al also found that when these AMPK activity enhancers were given in parallel with dexamethasone, a synergistic increase in these ubiquitin ligases were seen. ${ }^{72}$ Dexamethasone is a commonly used, potent inducer of experimental atrophy that highly up-regulates the ubiquitin ligases MuRF1 and MAFBx/

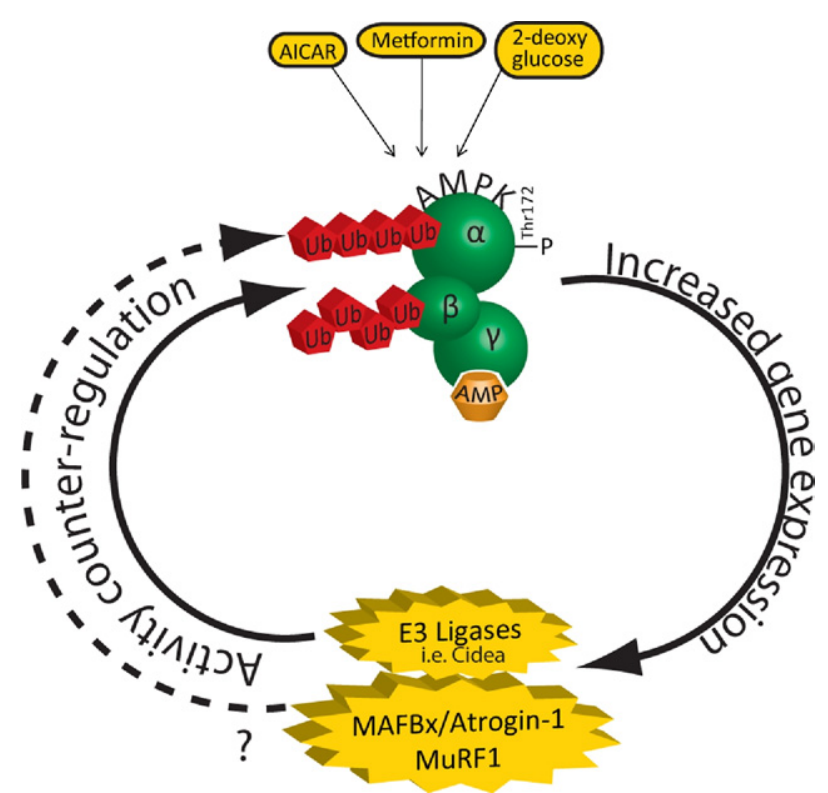

Figure 3. AMPK agonists enhance the expression of the ubiquitin ligases MAFBx/Atrogin-1 and MuRF1. Enhancing AMPK activity using AMPK agonists in C2C12 myotubes increases the expression of MAFBx/Atrogin-1 and MuRF1 (discussed in the text). With evidence that AMPK is ubiquitinated (outlined in Figure $2^{58}$ ), it raises the possible counterregulation of AMPK yet-to-be-determined E3 ligases, or possibly MAFBx/Atrogin-1 and MuRF1, although this remains to be investigated directly. 
atrogin-1 that directly mediate muscle atrophy. AICAR, metformin, and 2-deoxyglucose treatment (with and without dexamethasone treatment) did not enhance the expression of other UBR box E3 ubiquitin ligases, including UBR1/E3 alphal and UBR2/E3 alphall, suggesting that AMPK was specifically activating ubiquitin ligases that are known to mediate muscle atrophy. ${ }^{72}$ Inhibiting AICAR with compound C prevented MAFBx/atrogin-1 and MuRF1 expression increases in response to serum deprivation, AICAR treatment, or AICAR and dexamethasone treatment together..$^{72}$ These findings were also seen in in vivo models. When mice were challenged with AICAR, an increase in skeletal muscle MAFBx/atrogin-1 and MuRF1 mRNA was identified, suggesting a role of AMPK in the regulation of these ubiquitin ligases in vivo. Since these ubiquitin ligases are critical components of the complex network of signaling pathways activated in skeletal muscle atrophy, AMPK may play key roles in regulating atrophy in response to catabolic challenges.

Insulin-like growth factor 1 (IGF-1) stimulation enhances downstream signaling through activating (phosphorylating) Akt, which in turn phosphorylates the transcription factor FoxO3a, resulting in a reduction in the ubiquitin ligases MAFBx/atrogin-1 and MuRF1. ${ }^{73-75}$ Recent studies have identified that AICAR synergizes with IGF-1 induced Akt activation without affecting (inhibiting) the expression of the MAFBx/atrogin-1 and MuRF1 ubiquitin ligases. ${ }^{76}$ It was identified that AICAR inhibited Fox03a phosphorylation in the cytoplasm and induced Fox03a nuclear relocation. ${ }^{76}$ Inhibiting mTOR increased basal MAFBx/atrogin-1 expression and reversed the inhibitor effect of IGF-1 on ubiquitin ligase expression. These studies demonstrate that AICAR activation of AMPK stimulates MAFBx/atrogin-1and MuRF1 expression despite the activation of Akt signaling, which may be due to the inhibition of Fox0 phosphorylation by AMPK via Akt. ${ }^{76}$ AMPK inhibition of mTOR may additionally provide a mechanism by which AMPK enhances the expression of ubiquitin ligases. ${ }^{76}$

These studies uniformly demonstrate that enhancing AMPK activity increases the expression of ubiquitin ligases fundamentally involved in skeletal muscle and cardiac atrophy (Figure 3). And specific ubiquitin ligases ubiquitinate AMPK directly, such as Cidea (Figure 3). Whether there is a feedback loop by which the MuRF1 and MAFBX/Atrogin-1 ubiquitin ligases limit AMPK has not been determined. This is a logical possibility, given the several ways in which ubiquitination regulates AMPK, either directly or indirectly, as outlined in the previous section (Figure $2^{58}$ ). MuRF1 has been reported to have the ability to place atypical ubiquitin chains, like those found on AMPK experimentally. ${ }^{53}$ While these studies have been uniformly performed in skeletal muscle, the ubiquitin ligases MAFBx/atrogin-1 and MuRF1 have proven to have a significant role in cardiac disease (see recent review ${ }^{50}$ ). For example, MuRF1 inhibits cardiac hypertrophy, is necessary for regression of cardiac hypertrophy, and is cardioprotective in I/R injury by inhibiting transcription factors such as SRF and cJun. ${ }^{50}$ Similarly, MAFBx/atrogin-1 inhibits pressure-overload induced cardiac hypertrophy and increases susceptibility to $\mathrm{I} / \mathrm{R}$ induced apoptosis by proteasome-dependent degradation of MKP-1, a negative regulator of $\mathrm{JNK}^{50}$ With the diverse regulation of AMPK during various cardiac stresses (Table 1 14,22,26,36-49), it is certainly possible that AMPK upstream regulation of MAFBx/atrogin-1 and MuRF1 is involved in the heart's adaptive responses to injury. However, this remains to be directly tested.

\section{Conclusions}

The 5'-AMP-activated protein kinase (AMPK) functions as a metabolic fuel gauge activated in response to numerous environmental stressors to restore cellular energy balance. In the heart, AMPK coordinates the activation of glucose and fatty acid metabolic pathways at multiple levels by regulating substrate availability, uptake, and breakdown-ultimately ensuring increased production of myocardial ATP when required. Cardiac AMPK activity is regulated in cardiac diseases such as ischemia/reperfusion injury and is implicated in regulating cardiac metabolism to exert both positive and negative roles in cardiac ischemia/reperfusion injury and cardiac hypertrophy (Table $1^{14,22,26,36-49}$ ). Although the basic regulation of AMPK activity by kinases has been delineated, we are beginning to understand that other post-translational modifications may regulate AMPK activity as well. Recent reports determined that the ubiquitin ligase Cidea ubiquitinates the $\beta$ subunit of AMPK, leading to degradation. Atypical ubiquitination has been identified on the $\alpha$ subunit as well as two of the regulatory AMPK kinases NUAK1 and MARK4, which regulate AMPK activity. Activation of AMPK using agonists such as AICAR, metformin, and 2-deoxyglucose increases the expression of key cardiac ubiquitin ligases MAFBx/Atrogin-1 and MuRF1. Given the potential of these ubiquitin ligases to ubiquitinate key cardiac transcription factors as well as AMPK itself suggests that AMPK's role in cardiovascular biology extends beyond metabolism and is important in MAFBX/Atrogin-1- and MuRF1-dependent remodeling and cell signaling regulation. The relevance of the ubiquitination of AMPK in cardiac disease has not been tested directly, but likely represents an important mechanism that occurs in common cardiac diseases and that potentially could be targeted for therapy. How the ubiquitin proteasome system regulates AMPK activity is an emerging concept with far-reaching implications.

\section{References}

1. Hardie DG, Hawley SA, Scott JW: AMP-activated protein kinasedevelopment of the energy sensor concept. J Physiol 2006, 574:7-15

2. Young LH, Li J, Baron SJ, Russell RR: AMP-activated protein kinase: a key stress signaling pathway in the heart. Trends Cardiovasc Med 2005, 15:110-118

3. Hardie DG: Minireview: the AMP-activated protein kinase cascade: the key sensor of cellular energy status. Endocrinology 2003, 144 5179-5183

4. Dyck JR, Lopaschuk GD: AMPK alterations in cardiac physiology and pathology: enemy or ally? J Physiol 2006, 574:95-112

5. Kerscher O, Felberbaum R, Hochstrasser M: Modification of proteins by ubiquitin and ubiquitin-like proteins. Annu Rev Cell Dev Biol 2006 22:159-180 
6. Steinberg GR, Kemp BE: AMPK in Health and Disease. Physiol Rev 2009, 89:1025-1078

7. Woods A, Vertommen D, Neumann D, Turk R, Bayliss J, Schlattner U, Wallimann $\mathrm{T}$, Carling $\mathrm{D}$, Rider $\mathrm{MH}$ : Identification of phosphorylation sites in AMP-activated protein kinase (AMPK) for upstream AMPK kinases and study of their roles by site-directed mutagenesis. J Biol Chem 2003, 278:28434-28442

8. Polekhina G, Gupta A, Michell BJ, van Denderen B, Murthy S, Feil SC, Jennings IG, Campbell DJ, Witters LA, Parker MW, Kemp BE, Stapleton D: AMPK beta subunit targets metabolic stress sensing to glycogen. Curr Biol 2003, 13:867-871

9. Warden SM, Richardson C, O'Donnell J Jr, Stapleton D, Kemp BE, Witters LA: Post-translational modifications of the beta-1 subunit of AMP-activated protein kinase affect enzyme activity and cellular localization, Biochem J 2001, 354:275-283

10. Bateman A: The structure of a domain common to archaebacteria and the homocystinuria disease protein. Trends Biochem Sci 1997 22:12-13

11. Scott JW, Hawley SA, Green KA, Anis M, Stewart G, Scullion GA, Norman DG, Hardie DG: CBS domains form energy-sensing modules whose binding of adenosine ligands is disrupted by disease mutations. J Clin Invest 2004, 113:274-284

12. Stapleton D, Mitchelhill KI, Gao G, Widmer J, Michell BJ, Teh T, House CM, Fernandez CS, Cox T, Witters LA, Kemp BE: Mammalian AMP-activated protein kinase subfamily. J Biol Chem 1996, 271:611614

13. Thornton C, Snowden MA, Carling D: Identification of a novel AMPactivated protein kinase beta subunit isoform that is highly expressed in skeletal muscle. J Biol Chem 1998, 273:12443-12450

14. Arad M, Seidman CE, Seidman JG: AMP-activated protein kinase in the heart: role during health and disease. Circ Res 2007, 100:474488

15. Cheung PC, Salt IP, Davies SP, Hardie DG, Carling D: Characterization of AMP-activated protein kinase gamma-subunit isoforms and their role in AMP binding. Biochem J 2000, 346(Pt 3):659-669

16. Hardie DG: Role of AMP-activated protein kinase in the metabolic syndrome and in heart disease. FEBS Lett 2008, 582:81-89

17. Rosenfeldt F: Heart physiology from cell to circulation. Edited by LH Opie. Philadelphia, Lippincott Williams \& Wilkins, 2004, p. 154

18. Suter M, Riek U, Tuerk R, Schlattner U, Wallimann T, Neumann D: Dissecting the role of $5^{\prime}$-AMP for allosteric stimulation, activation, and deactivation of AMP-activated protein kinase. J Biol Chem 2006 , 281:32207-32216

19. Nagata D, Hirata Y: The role of AMP-activated protein kinase in the cardiovascular system. Hypertens Res 2010, 33:22-28

20. Davies SP, Helps NR, Cohen PT, Hardie DG: 5'-AMP inhibits dephosphorylation, as well as promoting phosphorylation, of the AMP-activated protein kinase. Studies using bacterially expressed human protein phosphatase-2C alpha and native bovine protein phosphatase-2AC. FEBS Lett 1995, 377:421-425

21. Anderson KA, Means RL, Huang QH, Kemp BE, Goldstein EG, Selbert MA, Edelman AM, Fremeau RT, Means AR: Components of a calmodulin-dependent protein kinase cascade. Molecular cloning functional characterization and cellular localization of $\mathrm{Ca} 2+/$ calmodulin-dependent protein kinase kinase beta. J Biol Chem 1998, 273 31880-31889

22. Sakamoto K, Zarrinpashneh E, Budas GR, Pouleur AC, Dutta A Prescott AR, Vanoverschelde JL, Ashworth A, Jovanovic A, Aless DR, Bertrand L: Deficiency of LKB1 in heart prevents ischemiamediated activation of AMPKalpha2 but not AMPKalpha1. Am $J$ Physiol Endocrinol Metab 2006, 290:E780-788

23. Gamble J, Lopaschuk GD: Insulin inhibition of $5^{\prime}$ adenosine monophosphate-activated protein kinase in the heart results in activation of acetyl coenzyme A carboxylase and inhibition of fatty acid oxidation. Metabolism 1997, 46:1270-1274

24. Kovacic S, Soltys CL, Barr AJ, Shiojima I, Walsh K, Dyck JR: Akt activity negatively regulates phosphorylation of AMP-activated protein kinase in the heart. J Biol Chem 2003, 278:39422-39427

25. Longnus SL, Segalen C, Giudicelli J, Sajan MP, Farese RV, Van Obberghen $\mathrm{E}$ : Insulin signalling downstream of protein kinase $\mathrm{B}$ is potentiated by $5^{\prime}$ AMP-activated protein kinase in rat hearts in vivo. Diabetologia 2005, 48:2591-2601

26. Shibata R, Ouchi N, Ito M, Kihara S, Shiojima I, Pimentel DR, Kumada M, Sato K, Schiekofer S, Ohashi K, Funahashi T, Colucci WS, Walsh
K: Adiponectin-mediated modulation of hypertrophic signals in the heart. Nat Med 2004, 10:1384-1389

27. Shimano M, Ouchi N, Shibata R, Ohashi K, Pimentel DR, Murohara T, Walsh K: Adiponectin deficiency exacerbates cardiac dysfunction following pressure overload through disruption of an AMPKdependent angiogenic response. J Mol Cell Cardiol 2010, 49: 210-220

28. Xie M, Zhang D, Dyck JR, Li Y, Zhang H, Morishima M, Mann DL, Taffet GE, Baldini A, Khoury DS, Schneider MD: A pivotal role for endogenous TGF-beta-activated kinase-1 in the LKB1/AMP-activated protein kinase energy-sensor pathway. Proc Natl Acad Sci U S A 2006, 103:17378-17383

29. Wong AK, Howie J, Petrie JR, Lang CC: AMP-activated protein kinase pathway: a potential therapeutic target in cardiometabolic disease. Clin Sci (Lond) 2009, 116:607-620

30. El-Mir MY, Nogueira V, Fontaine E, Averet N, Rigoulet M, Leverve X Dimethylbiguanide inhibits cell respiration via an indirect effect targeted on the respiratory chain complex I. J Biol Chem 2000, 275 223-228

31. Owen MR, Doran E, Halestrap AP: Evidence that metformin exerts its anti-diabetic effects through inhibition of complex 1 of the mitochondrial respiratory chain, Biochem J 2000, 348(Pt 3):607-614

32. Hawley SA, Gadalla AE, Olsen GS, Hardie DG: The antidiabetic drug metformin activates the AMP-activated protein kinase cascade via an adenine nucleotide-independent mechanism. Diabetes 2002, 51 $2420-2425$

33. Stanley WC, Recchia FA, Lopaschuk GD: Myocardial substrate metabolism in the normal and failing heart. Physiol Rev 2005, 85:10931129

34. Jorgensen SB, Richter EA, Wojtaszewski JF: Role of AMPK in skeletal muscle metabolic regulation and adaptation in relation to exercise. J Physiol 2006, 574:17-31

35. Dolinsky VW, Dyck JR: Role of AMP-activated protein kinase in healthy and diseased hearts. Am J Physiol Heart Circ Physiol 2006 , 291:H2557-2569

36. Lopaschuk GD: AMP-activated protein kinase control of energy metabolism in the ischemic heart. Int J Obes (Lond) 2008, 32(Supp 4):S29-35

37. Russell RR 3rd, Li J, Coven DL, Pypaert M, Zechner C, Palmeri M, Giordano FJ, Mu J, Birnbaum MJ, Young LH: AMP-activated protein kinase mediates ischemic glucose uptake and prevents postischemic cardiac dysfunction, apoptosis, and injury. J Clin Invest 2004 114:495-503

38. Altarejos JY, Taniguchi M, Clanachan AS, Lopaschuk GD: Myocardial ischemia differentially regulates LKB1 and an alternate 5'-AMP-activated protein kinase. J Biol Chem 2005, 280:183-190

39. Paiva MA, Goncalves LM, Providencia LA, Davidson SM, Yellon DM, Mocanu MM: Transitory activation of AMPK at reperfusion protects the ischaemic-reperfused rat myocardium against infarction. Cardiovasc Drugs Ther 2010, 24:25-32

40. Nishino Y, Miura T, Miki T, Sakamoto J, Nakamura Y, Ikeda Y, Kobayashi $\mathrm{H}$, Shimamoto $\mathrm{K}$ : Ischemic preconditioning activates AMPK in a PKC-dependent manner and induces GLUT4 up-regulation in the late phase of cardioprotection. Cardiovasc Res 2004, 61:610-619

41. Dyck JR, Kudo N, Barr AJ, Davies SP, Hardie DG, Lopaschuk GD: Phosphorylation control of cardiac acetyl-CoA carboxylase by CAMPdependent protein kinase and 5'-AMP activated protein kinase. Eur J Biochem 1999, 262:184-190

42. Essop MF, Opie LH: Metabolic therapy for heart failure, Eur Heart $J$ 2004, 25:1765-1768

43. Gollob MH: Glycogen storage disease as a unifying mechanism of disease in the PRKAG2 cardiac syndrome. Biochem Soc Trans 2003, $31: 228-231$

44. Arad M, Moskowitz IP, Patel VV, Ahmad F, Perez-Atayde AR, Sawyer DB, Walter M, Li GH, Burgon PG, Maguire CT, Stapleton D, Schmitt JP, Guo XX, Pizard A, Kupershmidt S, Roden DM, Berul Cl, Seidman CE, Seidman JG: Transgenic mice overexpressing mutant PRKAG2 define the cause of Wolff-Parkinson-White syndrome in glycogen storage cardiomyopathy. Circulation 2003, 107:2850-2856

45. Sidhu JS, Rajawat YS, Rami TG, Gollob MH, Wang Z, Yuan R, Marian AJ, DeMayo FJ, Weilbacher D, Taffet GE, Davies JK, Carling D, Khoury DS, Roberts R: Transgenic mouse model of ventricular preexcitation and atrioventricular reentrant tachycardia induced by an 
AMP-activated protein kinase loss-of-function mutation responsible for Wolff-Parkinson-White syndrome. Circulation 2005, 111:21-29

46. Arad M, Benson DW, Perez-Atayde AR, McKenna WJ, Sparks EA, Kanter RJ, McGarry K, Seidman JG, Seidman CE: Constitutively active AMP kinase mutations cause glycogen storage disease mimicking hypertrophic cardiomyopathy. J Clin Invest 2002, 109:357-362

47. Gollob MH, Seger JJ, Gollob TN, Tapscott T, Gonzales O, Bachinsk L, Roberts R: Novel PRKAG2 mutation responsible for the genetic syndrome of ventricular preexcitation and conduction system disease with childhood onset and absence of cardiac hypertrophy. Circulation 2001, 104:3030-3033

48. Tian R, Musi N, D'Agostino J, Hirshman MF, Goodyear LJ: Increased adenosine monophosphate-activated protein kinase activity in rat hearts with pressure-overload hypertrophy. Circulation 2001, 104: 1664-1669

49. Chan AY, Soltys CL, Young ME, Proud CG, Dyck JR: Activation of AMP-activated protein kinase inhibits protein synthesis associated with hypertrophy in the cardiac myocyte. J Biol Chem 2004, 279: 32771-32779

50. Rodriguez JE, Schisler JC, Patterson C, Willis MS: Seek and destroy: the ubiquitin-proteasome system in cardiac disease. Curr Hypertens Rep 2009, 11:396-405

51. Reyes-Turcu FE, Ventii KH, Wilkinson KD: Regulation and cellular roles of ubiquitin-specific deubiquitinating enzymes. Annu Rev Biochem 2009, 78:363-397

52. Kirisako T, Kamei K, Murata S, Kato M, Fukumoto H, Kanie M, Sano S, Tokunaga F, Tanaka K, Iwai K: A ubiquitin ligase complex assembles linear polyubiquitin chains. EMBO J 2006, 25:4877-4887

53. Kim HT, Kim KP, Lledias F, Kisselev AF, Scaglione KM, Skowyra D, Gygi SP, Goldberg AL: Certain pairs of ubiquitin-conjugating enzymes (E2s) and ubiquitin-protein ligases (E3s) synthesize nondegradable forked ubiquitin chains containing all possible isopeptide linkages. J Biol Chem 2007, 282:17375-17386

54. Ikeda F, Dikic I: Atypical ubiquitin chains: new molecular signals. "Protein modifications: beyond the usual suspects" review series. EMBO Rep 2008, 9:536-542

55. Haglund K, Dikic I: Ubiquitylation and cell signaling. EMBO J 2005 24:3353-3359

56. Hayden MS, Ghosh S: Shared principles in NF-kappaB signaling. Cell 2008, 132:344-362

57. Al-Hakim AK, Zagorska A, Chapman L, Deak M, Peggie M, Alessi DR: Control of AMPK-related kinases by USP9X and atypical Lys(29)/ Lys(33)-linked polyubiquitin chains. Biochem J 2008, 411:249-260

58. Witczak CA, Sharoff CG, Goodyear LJ: AMP-activated protein kinase in skeletal muscle: from structure and localization to its role as a master regulator of cellular metabolism. Cell Mol Life Sci 2008, 65: 3737-3755

59. Katz EJ, Isasa M, Crosas B: A new map to understand deubiquitination. Biochem Soc Trans 2010, 38:21-28

60. Shabek N, Ciechanover A: Degradation of ubiquitin: the fate of the cellular reaper. Cell Cycle, 2010, 9:523-530

61. Kimura Y, Tanaka K: Regulatory mechanisms involved in the control of ubiquitin homeostasis. J Biochem 2010, 147:793-798

62. Colland $F$ : The therapeutic potential of deubiquitinating enzyme inhibitors. Biochem Soc Trans, 38:137-143
63. Qi J, Gong J, Zhao T, Zhao J, Lam P, Ye J, Li JZ, Wu J, Zhou HM, Li $P$ : Downregulation of AMP-activated protein kinase by Cidea-mediated ubiquitination and degradation in brown adipose tissue. EMBO J 2008, 27:1537-1548

64. Chastagner P, Israel A, Brou C: Itch/AIP4 mediates Deltex degradation through the formation of K29-linked polyubiquitin chains. EMBO Rep 2006, 7:1147-1153

65. Moreno D, Viana R, Sanz P: Two-hybrid analysis identifies PSMD11, a non-ATPase subunit of the proteasome, as a novel interaction partner of AMP-activated protein kinase. Int J Biochem Cell Biol 2009, 41:2431-2439

66. Henin N, Vincent MF, Gruber HE, Van den Berghe G: Inhibition of fatty acid and cholesterol synthesis by stimulation of AMP-activated protein kinase. FASEB J 1995, 9:541-546

67. Park H, Kaushik VK, Constant S, Prentki M, Przybytkowski E, Ruderman NB, Saha AK: Coordinate regulation of malonyl-CoA decarboxylase, sn-glycerol-3-phosphate acyltransferase, and acetyl-CoA carboxylase by AMP-activated protein kinase in rat tissues in response to exercise. J Biol Chem 2002, 277:32571-32577

68. Bolster DR, Crozier SJ, Kimball SR, Jefferson LS: AMP-activated protein kinase suppresses protein synthesis in rat skeletal muscle through down-regulated mammalian target of rapamycin (mTOR) signaling. J Biol Chem 2002, 277:23977-23980

69. Dubbelhuis PF, Meijer AJ: Hepatic amino acid-dependent signaling is under the control of AMP-dependent protein kinase. FEBS Lett 2002 , 521:39-42

70. Horman S, Browne G, Krause U, Patel J, Vertommen D, Bertrand L, Lavoinne A, Hue L, Proud C, Rider M: Activation of AMP-activated protein kinase leads to the phosphorylation of elongation factor 2 and an inhibition of protein synthesis. Curr Biol 2002, 12:1419-1423

71. Nakashima K, Yakabe Y: AMPK activation stimulates myofibrillar protein degradation and expression of atrophy-related ubiquitin ligases by increasing FOXO transcription factors in $\mathrm{C} 2 \mathrm{C} 12$ myotubes. Biosci Biotechnol Biochem 2007, 71:1650-1656

72. Krawiec BJ, Nystrom GJ, Frost RA, Jefferson LS, Lang CH: AMPactivated protein kinase agonists increase mRNA content of the muscle-specific ubiquitin ligases MAFbx and MuRF1 in C2C12 cells. Am J Physiol Endocrinol Metab 2007, 292:E1555-1567

73. Sandri M, Sandri C, Gilbert A, Skurk C, Goldberg AL: Foxo transcription factors induce the atrophy-related ubiquitin ligase atrogin-1 and cause skeletal muscle atrophy. Cell 2004, 117:399-412

74. Stitt TN, Drujan D, Clarke BA, Panaro F, Timofeyva Y, Kline WO, Gonzalez M, Yancopoulos GD, Glass DJ: The IGF-1/PI3K/Akt pathway prevents expression of muscle atrophy-induced ubiquitin ligases by inhibiting FOXO transcription factors. Mol Cell 2004, 14:395-403

75. Lee SW, Dai G, Hu Z, Wang X, Du J, Mitch WE: Regulation of muscle protein degradation: coordinated control of apoptotic and ubiquitinproteasome systems by phosphatidylinositol 3 kinase. J Am Soc Nephrol 2004, 15:1537-1545

76. Tong JF, Yan X, Zhu MJ, Du M: AMP-activated protein kinase enhances the expression of muscle-specific ubiquitin ligases despite its activation of IGF-1/Akt signaling in C2C12 myotubes. J Cell Biochem 2009, 108:458-468 